\title{
Chest ultrasound for evaluation of bilateral pulmonary infiltrates in initensive care unit: a comparison with clinical assessment, sonographic assessment
}

\author{
J Jeon*, SB Hong \\ From ESICM LIVES 2015 \\ Berlin, Germany. 3-7 October 2015
}

\section{Background}

When the new bilateral air space pacification develops, we are usually indistinguishable bilateral pulmonary infiltrates from congestive cardiac failure. Until now, for the differential diagnosis of cardiogenic edema and pneumonia, laboratory finding including white blood cell (WBC), C-reactive protein (CRP), B-type natriuretic peptide (BNP) and imaging studies such as chest computed tomography $(\mathrm{CT})$ and/or echocardiography were have been used. We evaluated whether chest ultrasound (CUS, heart+lung) could assist in the differential diagnosis of pulmonary edema and pneumonia. So, we conducted a comparison between clinical diagnosis (C-Dx) with the result of the laboratory finding and sonographic diagnosis (US-Dx) with CUS.

\section{Method \\ We performed a retrospective observational study evalu- ating the utility of CUS in helping to differentiate pul- monary edema and pneumonia who were admitted to the ICU from January 2013 to December 2014. The patients who admitted to the medical intensive care unit (MICU), of the patients showed new developed bilateral infiltra- tion on chest X-ray, targeting patients who underwent CUS. CUS was performed in all patients on the first day of admission and after having obtained a clinical diagno- sis of disease. We compared the US-Dx is determined based on the cardiac and lung ultrasound with C-Dx is determined on the basis of WBC, CRP, BNP. WBC > $11,000 / \mathrm{uL}, \mathrm{CRP}>1 \mathrm{mg} / \mathrm{dL}$ and $\mathrm{BNP}>300 \mathrm{pg} / \mathrm{mL}$ were}

interpreted as a meaningful. C-Dx and US-Dx were compared to see if it matches divided into three categories of pneumonia, pulmonary edema, combined.

\section{Result}

During the 2-years study period, seventy-seven patients were enrolled. C-Dx was associated with US-Dx $(\mathrm{P}<$ 0.00 , Fisher's exact test). US-Dx was associated with final diagnosis $(\mathrm{P}<0.00$, Fisher's exact test).

\section{Conclusion}

In critically ill patients, diagnosis using chest ultrasound and diagnosis was inferred to be the result of laboratory test substantially coincided. Therefore, using such a point, it is useful for the differential diagnosis of the patient.

Published: 1 October 2015

doi:10.1186/2197-425X-3-S1-A613

Cite this article as: Jeon and Hong: Chest ultrasound for evaluation of bilateral pulmonary infiltrates in initensive care unit: a comparison with clinical assessment, sonographic assessment. Intensive Care Medicine Experimental 2015 3(Suppl 1):A613. 